\title{
Histone deacetylase inhibitor givinostat alleviates liver fibrosis by regulating hepatic stellate cell activation
}

\author{
HE-MING HUANG ${ }^{1,2^{*}}$, XIAO-RU ZHOU ${ }^{2 *}$, YAN-JUN LIU $^{1,2^{*}}$, SHI-JIE FAN $^{2,3}$, LI-PING LIAO $^{2,3}$, JING HUANG $^{2,3}$, \\ CUI-CUI SHI ${ }^{1}$, LIANG YU ${ }^{2}$, JIN-JIN PEN ${ }^{1,2}$, CHENG LUO $^{2,3}$, YUAN-YUAN ZHANG ${ }^{2}$ and GUANG-MING LI ${ }^{1}$ \\ ${ }^{1}$ Department of Gastroenterology, Xinhua Hospital, School of Medicine, Shanghai Jiaotong University, \\ Shanghai 200092; ${ }^{2}$ Drug Discovery and Design Center, State Key Laboratory of Drug Research, \\ Shanghai Institute of Materia Medica, Chinese Academy of Sciences, Shanghai 201203; \\ ${ }^{3}$ Chemical Biology Center, University of Chinese Academy of Sciences, Beijing 100049, P.R. China
}

Received July 17, 2020; Accepted January 8, 2021

DOI: $10.3892 / \mathrm{mmr} .2021 .11944$

\begin{abstract}
Hepatic fibrosis, a common pathological manifestation of chronic liver injury, is generally considered to be the end result of an increase in extracellular matrix produced by activated hepatic stellate cells (HSCs). The aim of the present study was to target the mechanisms underlying HSC activation in order to provide a powerful therapeutic strategy for the prevention and treatment of liver fibrosis. In the present study, a high-throughput screening assay was established, and the histone deacetylase inhibitor givinostat was identified as a potent inhibitor of HSC activation in vitro. Givinostat significantly inhibited HSC activation in vivo, ameliorated carbon tetrachloride-induced mouse liver fibrosis and lowered plasma aminotransferases. Transcriptomic analysis revealed the most significantly regulated genes in the givinostat treatment group in comparison with those in the solvent group, among which, dermokine (Dmkn), mesothelin (Msln) and uroplakin-3b (Upk3b) were identified as potential regulators of HSC activation. Givinostat significantly reduced the mRNA expression of $D m k n, M s l n$ and $U p k 3 b$ in both a mouse liver fibrosis model and in HSC-LX2 cells. Knockdown of any of the aforementioned
\end{abstract}

Correspondence to: Dr Guang-Ming Li or Dr Cui-Cui Shi, Department of Gastroenterology, Xinhua Hospital, School of Medicine, Shanghai Jiaotong University, 1665 Kongjiang Road, Shanghai 200092, P.R. China

E-mail: liguangming@xinhuamed.com.cn

E-mail: shicuicui2005@126.com

*Contributed equally

Abbreviations: HSCs, hepatic stellate cells; ECM, extracellular matrix; CCl4, carbon tetrachloride; $\alpha$-SMA, $\alpha$-smooth muscle actin; Coll $\alpha 1$, collagen type I; HDAC, histone deacetylase; HDACi, HDAC inhibitor; ALT, alanine aminotransferase; AST, aspartate aminotransferase

Key words: liver fibrosis, hepatic stellate cell, histone deacetylase inhibitor, epigenetics, givinostat genes inhibited the TGF- $\beta 1$-induced expression of $\alpha$-smooth muscle actin and collagen type I, indicating that they are crucial for HSC activation. In summary, using a novel strategy targeting HSC activation, the present study identified a potential epigenetic drug for the treatment of hepatic fibrosis and revealed novel regulators of HSC activation.

\section{Introduction}

Cirrhosis is an increasing global health burden that accounts for $>100$ million deaths annually worldwide (1). Liver fibrosis is the result of wound-healing response to chronic liver impairment triggered by a variety of causes, including hepatitis virus, ethanol, drugs and poisons, parasites, metabolism and genetics, cholestasis and immune deregulation $(2,3)$. Without diagnosis and treatment, hepatic fibrosis will ultimately progress to hepatic cirrhosis, and even to hepatocellular carcinoma (4). Thus, it is of great importance to actively intervene in liver fibrosis. Hepatic fibrosis is characterized by the deposition of extracellular matrix (ECM) proteins, which destroy the normal liver histological structure and functions (5). Hepatic stellate cells (HSCs) play a vital role in the development of liver fibrosis, and are the main producers of ECM (3). In the case of liver injury, certain cytokines and growth factors crucial for HSC activation are released, and promote HSC activation into myofibroblasts, which are responsible for the synthesis of ECM proteins, including $\alpha$-smooth muscle actin ( $\alpha$-SMA, which is encoded by Acta2), collagen type I (Col1 1 1), matrix metalloproteinases and tissue inhibitor of metalloproteinases (6). Therefore, directly inactivating HSCs is of great importance for fibrosis resolution, representing a therapeutic strategy for the treatment of hepatic fibrosis.

Epigenetic modifications regulate patterns of gene expression by modulating DNA accessibility and chromatin structure. The epigenetic machinery, particularly certain epigenetic enzymes, has been demonstrated to be involved in myofibroblast activation and regulation of fibrotic gene expression $(7,8)$. Blocking the expression of the DNA methyltransferase DNMT3B has been reported to significantly reduce $\alpha$-SMA and Col1 $\alpha 1$ expression in ischemic heart disease (9). In addition, the histone deacetylase (HDAC) 
inhibitors (HDACis), MS-275 and trichostatin A have been found to reduce renal fibrosis by diminishing the accumulation of ECM proteins (10-12). By contrast, other studies have indicated that targeted inhibition of certain epigenetic enzymes might aggravate fibrosis. It has been reported that inhibition of type I protein arginine methyltransferases can aggravate renal fibrosis by reducing asymmetric dimethylarginine accumulation, increasing nitric oxide concentrations and enhancing the expression of profibrotic proteins (13). However, there is currently no effective high-throughput screening method to identify candidate compounds for the treatment and prevention of liver fibrotic diseases.

Aiming to identify a novel candidate compound for the treatment of hepatic fibrosis, the present study established a cell-based high-throughput assay based on HSC activation, and screened our in-house epigenetic compound library (14). The HDACi givinostat, which has been used in phase I/II clinical trials for the treatment of Duchenne muscular dystrophy (15), was identified as the most potent hit. Givinostat reduced the expression of $\alpha$-SMA and collagen, which are markers of HSC activation in vitro. Carbon tetrachloride (CCl4) has been widely used to induce liver injury and fibrosis in mice for decades (16), and C57BL/6J inbred mice are frequently used for fibrosis studies in the $\mathrm{CCl} 4$ model of the ready availability of genetically-modified mice (17). In a chronic CCl4-challenged mouse model in the present study, mice developed mild liver fibrosis after 2 weeks of $\mathrm{CCl} 4$ treatment, and were then treated with givinostat for 6 weeks. Givinostat significantly ameliorated CCl4-induced mouse liver injury and fibrosis. RNA-sequencing (RNA-seq) analysis of the liver tissue from the givinostat treatment and solvent groups of CCl4-challenged mice revealed genes regulated by givinostat treatment, among which, dermokine (Dmkn), mesothelin $(M s l n)$ and uroplakin-3b $(U p k 3 b)$ were further identified as crucial genes regulating HSC activation. Givinostat inhibited HSC activation and alleviated liver fibrosis in vivo and in vitro, making it a promising tool for developing a novel therapy for the treatment of hepatic fibrosis.

\section{Materials and methods}

Animals and treatment. Female C57BL/6J mice (8-9 weeks old, weighting 21-23 g, specific pathogen-free) were purchased from the Animal Center of the Chinese Academy of Medical Sciences. Animal care was carried out according to the guidelines of the Principles of Laboratory Animal Care (18), all experimental protocols were approved by the Institutional Animal Care and Use Committee at the Shanghai Institute of Materia Medica (approval no. 2018-12-LC-11; Shanghai, China). The animals were allowed free access to a standard laboratory diet and tap water. All mice were kept in standard laboratory conditions $\left(21 \pm 2^{\circ} \mathrm{C}, 12\right.$-h light/dark cycle), and were fed adaptively for 1 week before starting the experiments. A total of 24 mice were randomly divided into three groups with 8 mice per group: i) The normal control group; ii) the solvent group of CCl4-challenged mice; and iii) the givinostat treatment group of $\mathrm{CCl} 4$-challenged mice. In the givinostat treatment group, mice were stimulated with $\mathrm{CCl} 4$ for 8 weeks, and in the last 6 weeks, the animals received an intraperitoneal (i.p.) injection of givinostat $(10 \mathrm{mg} / \mathrm{kg}$, formulated in PBS) daily (19). Mice were i.p. injected with $10 \% \mathrm{CCl} 4$ dissolved in olive oil at a dose of $1 \mathrm{ml} / \mathrm{kg}$ body weight twice a week for 8 weeks to trigger liver fibrosis (20). Givinostat or PBS solvent was i.p. injected after $\mathrm{CCl} 4$ treatment for 2 weeks, when mild fibrosis was shown. At the end of the experiment, the mice were sacrificed, and blood as well as liver samples were harvested. Although there was a total of 24 mice used overall, too little blood was collected during blood collection to be used for experiments so the number of experimental results displayed was $n=8$ in normal control group, $n=6$ in $\mathrm{CCl} 4$ group and $\mathrm{n}=7$ in the $\mathrm{CCl} 4+$ givinostat group. All surgeries (blood and liver samples were harvested) were performed under sodium pentobarbital anesthesia $(50 \mathrm{mg} / \mathrm{kg})$, and then all mice were euthanized by $5 \%$ isoflurane (cat. no. HR135327; Hairui Chemical). Death of the mice was confirmed by checking whether their heartbeat had completely stopped and whether their pupils were dilated.

Liver histopathology and immunohistochemistry. Liver tissues were fixed in $4 \%$ paraformaldehyde for $24 \mathrm{~h}$ at $37^{\circ} \mathrm{C}$, dehydrated and paraffin embedded. The 3-4-mm thick liver sections were stained with hematoxylin and eosin (cat. no. G1005; Wuhan Servicebio Technology Co., Ltd.) at $37^{\circ} \mathrm{C}$ for $5 \mathrm{~min}$ and $15 \mathrm{sec}$, respectively and Sirius Red (cat. no. G1018; Wuhan Servicebio Technology Co., Ltd.). The liver tissue sections were deparaffinized using xylene (Wuhan Servicebio Technology Co., Ltd.), rehydrated with graded alcohol, treated with $0.3 \%$ endogenous peroxidase blocking solution (Sigma-Aldrich; Merck KGaA) for $10 \mathrm{~min}$. Following high pressure heating retrieval $\left(125^{\circ} \mathrm{C}\right.$ and $103 \mathrm{kPa}$ ) and blocking with $10 \%$ normal goat serum (Wuhan Servicebio Technology Co., Ltd.) at $37^{\circ} \mathrm{C}$ for $30 \mathrm{~min}$, the sections were incubated overnight at $4^{\circ} \mathrm{C}$ with the following primary antibodies (Wuhan Servicebio Technology Co., Ltd.): anti- $\alpha$-SMA (cat. no. GB13044; 1:100) and anti-Col $1 \alpha 1$ (cat. no. GB11022-1; 1:100). Following washing with PBS, goat anti-rabbit non-biotinylated reagents (cat. no. G1213; 1:1,000; Wuhan Servicebio Technology Co., Ltd.) were used to react with the primary antibody for $2 \mathrm{~h}$ at $37^{\circ} \mathrm{C}$. Images were captured by observers who were blinded to the experimental conditions at 6-8 non-consecutive random fields under a light microscope (magnification, x100), and were used to assess the histological changes using Image-Pro Plus 6.0 software (Media Cybernetics, Inc.). Representative views were displayed.

Cell culture. The human HSC LX-2 cell line and the rat HSC-T6 cell line were obtained from the FuHeng Cell Center, and were cultured in Dulbecco's modified Eagle's medium (DMEM cat. no. L110KJ; Shanghai BasalMedia Technologies Co., Ltd.) supplemented with $10 \%$ fetal bovine serum (Gibco; Thermo Fisher Scientific, Inc.) and $1 \%$ penicillin and streptomycin (Gibco; Thermo Fisher Scientific, Inc.), at $37^{\circ} \mathrm{C}$ in a $95 \%$ air humidified atmosphere containing $5 \% \mathrm{CO}_{2}$. For stimulation, the cells were starved in serum-free DMEM for $24 \mathrm{~h}$ before being treated with recombinant human TGF- $\beta 1$ (10 ng/ml; cat. no. 100-21C; PeproTech, Inc.) (21) and/or givinostat $(900,300$ or $100 \mathrm{nM}$; cat. no. CSN16577; CSNpharm) for $24 \mathrm{~h}$.

One-step reverse transcription-quantitative PCR (RT-qPCR). HSC LX-2 cells $\left(5 \times 10^{5}\right.$ cells/well) were seeded in 96-well cell 
culture plates (Cellvis) overnight and sequentially stimulated with TGF- $\beta 1$ and compounds $(2 \mu \mathrm{M})$ or with TGF- $\beta 1$ solely. Cells were harvested $24 \mathrm{~h}$ after TGF- $\beta 1$ stimulation with Total RNA extraction reagent (Vazyme Biotech Co., Ltd.). Cytolysis was then subjected to RT-qPCR using TransScript ${ }^{\circledR}$ Green One-Step qRT-PCR SuperMix (cat. no. AQ211; TransGen Biotech Co., Ltd.) (14). The compounds library containing 46 molecule probes targeting epigenetic proteins was screened to find small molecule compounds able to inhibit $\alpha-S M A$ expression.

RNA-seq analysis. Total RNA was isolated from flash-frozen mice liver tissues. Total RNA was isolated and purified using DNase I (Takara Bio, Inc.) and Dynabeads Oligo (dT) 25 (Thermo Fisher Scientific, Inc.). Subsequently, purified RNA (100 ng) was used for cDNA library construction, using the NEBNext Ultra ${ }^{\text {TM }}$ RNA Library Prep kit for Illumina ${ }^{\circledR}$ (cat. no. E7530L; New England BioLabs, Inc.). Sequencing data was collected on an Illumina HiSeq 2500 instrument. The RNA integrity number (RIN) value was used to assess the quality of the isolated RNAs. Only RNAs with RIN $\geq 7.0$ were used for sequencing. The sequencing reads were located to mm10 by STAR 2.5 (22), and gene counting was quantified using featureCounts (Subread package 2.0.0) (23). The edgeR R package (24) was used for differential gene expression analysis. The P-value was adjusted using the Benjamini and Hochberg method (25), and a 5\% FDR cutoff value and fold-change $>1.5$ were set as the threshold of the significant gene. The differentially expressed genes were further analyzed by gene-annotation enrichment analysis using The Database for Annotation, Visualization and Integrated Discovery 6.8 bioinformatics platform (26). Cytoscape was used for network analysis (27). The original data generated using high-throughput sequencing methodologies has been submitted to the GEO database (https://www.ncbi.nlm.nih. gov/geo/query/acc.cgi?acc=GSE161981).

Small interfering (si)RNA transfection. Msln siRNA (sense, 5'-GCCUUGCUUUCCAGAACAU-3' and antisense, 5'-AUG UUCUGGAAAGCAAGGC-3'; and sense, 5'-GGACGUCCU AAAGCAUAAA-3' and antisense, 5'-UUUAUGCUUUAG GACGUCC-3'), Dmkn siRNA (sense, 5'-GCAGAGACGAUC AGAACUA-3' and antisense, 5'-UAGUUCUGAUCGUCU CUGC-3'; and sense, 5'-GCCUAUGGUGGGAAGUACU-3' and antisense, 5'-AGUACUUCCCACCAUAGGC-3') and Upk3b siRNA (sense, 5'-GCCCUACACACCACAGAUA-3' and antisense, 5'-UAUCUGUGGUGUGUAGGGC-3'; and sense, 5'-GCUACAUGACCCACCACAU-3' and antisense, 5'-AUGUGGUGGGUCAUGUAGC-3') for human cells were synthesized by Shanghai GenePharma Co., Ltd. Transfection with siRNA against Msln, Upk3b or Dmkn, or with control siRNA (sense, 5'-UUCUCCGAACGUGUCACGU-3' and antisense, 5'-ACGUGACACGUUCGGAGAA-3') was performed according to the manufacturer's protocol. LX-2 cells were seeded in a 6-well plate at $60-80 \%$ confluence. Briefly, siRNA $(20 \mu \mathrm{M}, 1.5 \mu \mathrm{l})$ and $9 \mu \mathrm{l}$ Lipofectamine ${ }^{\circledR}$ RNAiMAX transfection reagent (Invitrogen; Thermo Fisher Scientific, Inc.) were mixed with $150 \mu 1$ Opti MEM (cat. no. 31985070; Gibco; Thermo Fisher Scientific, Inc.). Next, diluted siRNA was added to diluted Lipofectamine RNAiMAX reagent and cultured for $5 \mathrm{~min}$ at room temperature. siRNA-lipid complex was added to cells for $6-8 \mathrm{~h}$ at $37^{\circ} \mathrm{C}$. Subsequent experiments were performed $24 \mathrm{~h}$ after transfection.

$R N A$ extraction and $R T-q P C R$. Total RNA was extracted from HSC LX-2 cells, HSC-T6 cells or liver tissues using TRIzol ${ }^{\circledR}$ reagent (Invitrogen; Thermo Fisher Scientific, Inc.), according to the manufacturer's protocols. Total RNA (1,000 ng) was reverse transcribed into cDNA using a cDNA synthesis kit (Vazyme Biotech Co., Ltd.). The reverse transcription temperature protocol was as follows: $50^{\circ} \mathrm{C}$ for $15 \mathrm{~min}$, followed by $80^{\circ} \mathrm{C}$ for 5 sec. RT-qPCR was performed using SYBR-Green (Vazyme Biotech Co., Ltd.). The thermocycling conditions were as follows: Pre-denaturation at $95^{\circ} \mathrm{C}$ for $10 \mathrm{~min}$, 40 cycles of denaturation at $95^{\circ} \mathrm{C}$ for $15 \mathrm{sec}$, and annealing at $60^{\circ} \mathrm{C}$ for $30 \mathrm{sec}$, followed by extension at $72^{\circ} \mathrm{C}$ for $1 \mathrm{~min}$. Subsequently, the expression values of mRNA were calculated using the $2^{-\Delta \Delta \mathrm{Cq}}$ method (28) The expression of target genes was normalized to GAPDH expression. The primer sequences are shown in Table I.

Biochemical analysis. The levels of serum aspartate aminotransferase (AST; cat. no. C010-3-1) and alanine aminotransferase (ALT; cat. no. C009-3-1) were measured using the corresponding kits (Nanjing Jiancheng Bioengineering Institute) and were assessed using a Hitachi 7020 automatic analyzer (Hitachi, Ltd.).

Western blotting. Total protein was extracted from HSC LX-2 cells, HSC-T6 cells or liver tissues with $1 \mathrm{X}$ SDS sample loading buffer $(250 \mathrm{mM}$ Tris HCL pH 6.8, 10\% SDS, 30\% glycerol, $5 \% \beta$-mercaptoethanol and $0.02 \%$ bromophenol blue). Protein concentration was determined using a BCA kit (Thermo Fisher Scientific, Inc.). The lysates (25 $\mu \mathrm{g} /$ lane) were separated via SDS-PAGE on 6, 10 or $12 \%$ gels, and subsequently transferred to a nitrocellulose membrane (EMD Millipore). Following blocking with $5 \%$ milk at room temperature for $1 \mathrm{~h}$, membranes were incubated with primary antibodies at $4^{\circ} \mathrm{C}$ overnight. Subsequently, the membranes were incubated with a HRP-conjugated goat anti-Rabbit IgG secondary antibody (1:10,000; cat. no. D110058; Sangon Biotech Co., Ltd.) for $1 \mathrm{~h}$ at room temperature. The bands were visualized using an enhanced chemiluminescence kit (Thermo Fisher Scientific, Inc.) with a ChemiScope 3400 mini imaging system (Clinx Science Instruments Co., Ltd.). Densitometry was performed for each group using ImageJ software (v1.50b; National Institutes of Health). The following primary antibodies were used: Anti- $\alpha$-SMA (1:1,000; cat. no. 19245; Cell Signaling Technology, Inc.), anti-Col1 $\alpha 1$ (1:1,000; cat. no. 72026; Cell Signaling Technology, Inc.) and anti-GAPDH $(1: 5,000$; cat. no. 8884; Cell Signaling Technology, Inc.), which was used as the loading control.

Statistical analysis. All numerical results are expressed as the mean \pm standard deviation, and represent data from a minimum of three independent experiments. Two-tailed unpaired t-test was used to analyze differences between two groups. One-way ANOVA or two-way ANOVA were used to compare the means of multiple groups followed by LSD post hoc test. All analyses were performed using GraphPad 
Table I. RT-qPCR primer sequences used in the present study.

A, Primer sequences used for mice liver tissues

\begin{tabular}{|c|c|}
\hline Gene & Primer sequences $\left(5^{\prime} \rightarrow 3^{\prime}\right)$ \\
\hline $\operatorname{Acta2}(\alpha-S M A)$ & $\begin{array}{l}\text { F: GCTGAAGTATCCGATAGAACACG } \\
\text { R: GGTCTCAAACATAATCTGGGTCA }\end{array}$ \\
\hline Coll $\alpha 1$ & $\begin{array}{l}\text { F: TCAGAGGCGAAGGCAACAGT } \\
\text { R: CCCCAAGTTCCGGTGTGA }\end{array}$ \\
\hline$G A P D H$ & $\begin{array}{l}\text { F: GGTGAAGGTCGGTGTGAACGGA } \\
\text { R: CCAAAGTTGTCATGGATGACCTTGG }\end{array}$ \\
\hline Sult3al & $\begin{array}{l}\text { F: TATTTTGAGGGTCATCGGAACAG } \\
\text { R: GGTGATGGCATTTTGGCATAGT }\end{array}$ \\
\hline$U p k 3 b$ & $\begin{array}{l}\text { F: CATCTGGCTAGTGGTGGCTTT } \\
\text { R: GGTAATGTCATATAGTGGCCGTC }\end{array}$ \\
\hline Adgrd1 & $\begin{array}{l}\text { F: GTCTGCTCTAGTGGTGGAAAGG } \\
\text { R: TAAGGACCGGGAGGGTTGAAG }\end{array}$ \\
\hline Snai3 & $\begin{array}{l}\text { F: GGTCCCCAACTACGGGAAAC } \\
\text { R: CTGTAGGGGGTCACTGGGATT }\end{array}$ \\
\hline$M \sin$ & $\begin{array}{l}\text { F: CACCGACGAGGAACTGAATG } \\
\text { R: CTCCGTGGGAGTACATCCACA }\end{array}$ \\
\hline Dmkn & $\begin{array}{l}\text { F: AGCTGACCAGTTTTCTAAGCC } \\
\text { R: GCCAGTTGTAAGTAGGATTCACC }\end{array}$ \\
\hline Upklb & $\begin{array}{l}\text { F: AACAGGAAAATCCTCTTGGCG } \\
\text { R: AAAAAGTCGCGTTGTGTTGCT }\end{array}$ \\
\hline Cebpe & $\begin{array}{l}\text { F: ATTCGCCTATCCCTCACACAC } \\
\text { R: GTAGCTGCCTCGACTGGTG }\end{array}$ \\
\hline Eif4ebp3 & $\begin{array}{l}\text { F: GTCCACGAGTTGCCCAATTC } \\
\text { R: GGGGTAGTGGCGTATAGTGTG }\end{array}$ \\
\hline Slc $2 a 5$ & $\begin{array}{l}\text { F: TTCCAATATGGGTACAACGTAGC } \\
\text { R: GCGTCAAGGTGAAGGACTCAA }\end{array}$ \\
\hline Ntrk1 & $\begin{array}{l}\text { F: GCCTAACCATCGTGAAGAGTG } \\
\text { R: CCAACGCATTGGAGGACAGAT }\end{array}$ \\
\hline
\end{tabular}

B, Primer sequences used for human HSC LX-2 cells

\begin{tabular}{ll}
\hline Gene & \multicolumn{1}{c}{ Primer sequences $\left(5^{\prime} \rightarrow 3^{\prime}\right)$} \\
\hline Acta2 $(\alpha-S M A)$ & F: TACGAGTAGAACGCTGTCCG \\
& R: CAGCACCGCCTGGATAGCC \\
Coll $\alpha 1$ & F: GTGCGATGACGTGATCTGTGA \\
& R: CGGTGGTTTCTTGGTCGGT \\
GAPDH & F: AGGTCGGTGTGAACGGATTTG \\
& R: GGGGTCGTTGATGGCAACA \\
SULT3A1 & F: CATTATAAAAGATGAACTCGG \\
& R: GAAAACAATCTAGGCACA \\
FPK3B & F: GCCTCTCCTCTGCCTTTCTGG \\
RDGRD1 & F: CTGCCTACTCAAATCTCTCTGC \\
& R: GAGTCCAATAGGGGCCTTCA \\
FNAI3 & F: CCCTTCCAAGAGTCAGAGCC \\
& R: TGCTGCAATGGAACTAGGCA
\end{tabular}

Table I. Continued.

B, Primer sequences used for human HSC LX-2 cells

\begin{tabular}{ll}
\hline Gene & \multicolumn{1}{c}{ Primer sequences $\left(5^{\prime} \rightarrow 3^{\prime}\right)$} \\
\hline$M S L N$ & F: CAGAGGAGGCTCAGAGAGCTA \\
& R: GTCCCACAGGACCCCAACAG \\
FMKN & F: CCAAGGGACCAGAGAAGCAG \\
& R: CCCAGTGTTTCCCAGAGCAT \\
FPK1B & F: GAACCTCTCAACCTGGAGGC \\
& R: TGGTACCCAGGAGAACCCAA \\
CEBPE & F: CTCCGATCTCTTTGCCGTGA \\
& R: GTCTGGGCCGAAGGTATGTG \\
FIF 4 F 32 CCACTAGCTGCCCGATTCC & R: GGTAGTGGCGTATAGCGTGC \\
& F: CAAGAAAGTTGAGTATGTTGGCT \\
NTRK1 & R: CAAGAAAGTTGAGTATGTTGGCT \\
& F: CCATCCCTGACACTAACAGCA \\
& R: GCACAAGGAGCAGCGTAGAA
\end{tabular}

F, forward, R, reverse; HSC, hepatic stellate cell; RT-qPCR, reverse transcription-quantitative PCR; $\alpha$-SMA, $\alpha$-smooth muscle actin; Col1 $\alpha 1$, collagen type I; Dmkn, dermokine; Msln, mesothelin; Upk3b, uroplakin-3b.

Prism 7.0 statistical software (GraphPad Software, Inc.). $\mathrm{P}<0.05$ was considered to indicate a statistically significant difference.

\section{Results}

Identification of givinostat as an inhibitor of HSC activation using cell-based high-throughput screening. Aiming to find potential candidate compounds that can inhibit HSC activation for the treatment of liver fibrosis, the present study established a high-throughput screening assay based on one-step RT-qPCR for detecting $\alpha$-SMA mRNA expression as a readout for HSC activation, since activated HSCs are characterized by the expression of $\alpha$-SMA (21). A library of small molecule inhibitors targeting epigenetic enzymes was screened, and givinostat was identified as the most potent compound that inhibited TGF- $\beta 1$-induced $\alpha$-SMA expression (Fig. 1A). Givinostat, a pan-HDACi that belongs to the hydroxamic acids family with HDAC type I and type II inhibitory activity, has been used in phase I/II clinical trials for the treatment of Duchenne muscular dystrophy. To validate the inhibitory effect of givinostat on HSC activation, the mRNA and protein expression levels of Coll $\alpha 1$ and $\alpha$-SMA after givinostat treatment were examined. Givinostat dose-dependently inhibited Coll $\alpha 1$ and $\alpha-S M A$ mRNA expression in the presence of TGF- $\beta 1$ (Fig. 1B). Consistently, western blotting also confirmed that givinostat inhibited Coll $\alpha 1$ and $\alpha$-SMA protein expression levels in a dose-dependent manner (Fig. 1C). Based on these data, givinostat was identified as a potent inhibitor of HSC activation in vitro. 

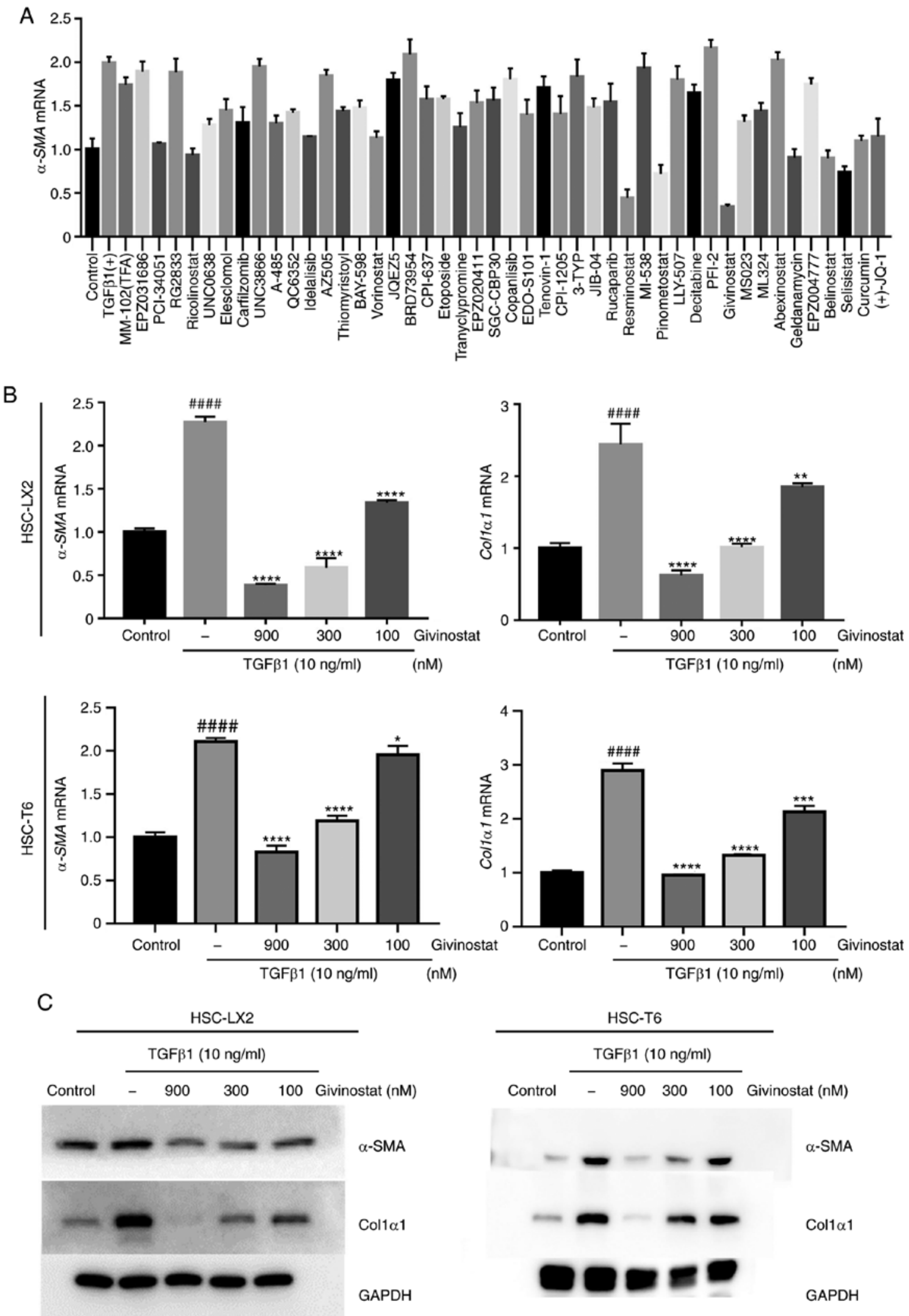

Figure 1. Givinostat treatment alleviates HSC activation in vitro. (A) Results of high-throughput screening using a library containing 46 small-molecule probes. (B) Reverse transcription-quantitative PCR analysis of the expression of $\alpha-S M A$ and Coll $\alpha 1$ after givinostat treatment at different concentrations in HSC LX-2 or HSC-T6 cells. (C) Western blot analyses of $\alpha$-SMA and Coll $\alpha 1$ protein levels in HSC LX-2 or HSC-T6 cells. The results are normalized to $\beta$-actin or GAPDH, and the error bars indicate the SD of three independent experiments. Data are expressed as the mean $\pm \mathrm{SD} .{ }^{*} \mathrm{P}<0.05,{ }^{* *} \mathrm{P}<0.01,{ }^{* * *} \mathrm{P}<0.001$,

${ }^{* * * * * *} \mathrm{P}<0.0001$ vs. the TGF- $\beta 1$ group; ${ }^{\# \# \# \#} \mathrm{P}<0.0001$ vs. the control group. HSC, hepatic stellate cell; $\alpha$-SMA, $\alpha$-smooth muscle actin; Coll $\alpha 1$, collagen type I.

Givinostat treatment alleviates chronic liver injury and fibrosis in mice treated with CCl4. Whether givinostat could inhibit HSC activation in vivo and alleviate liver fibrosis, which has not been extensively studied, was further examined 
A

Control 0

$\mathrm{CCl} 4$

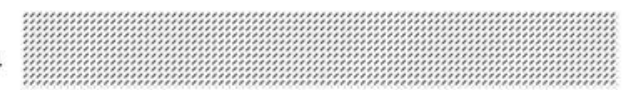

0

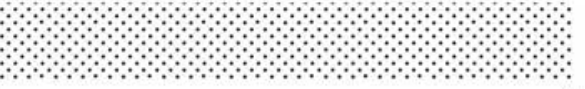

8 weeks

8 weeks

CCl4+givinostat

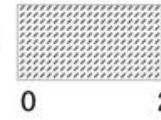

B Control

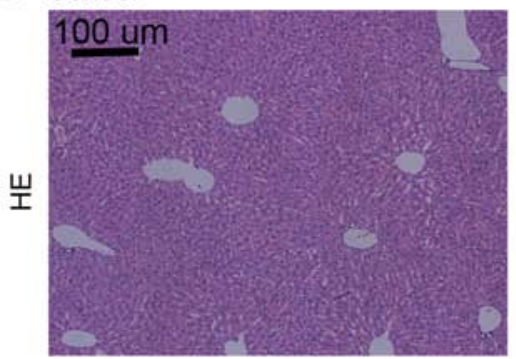

C Control

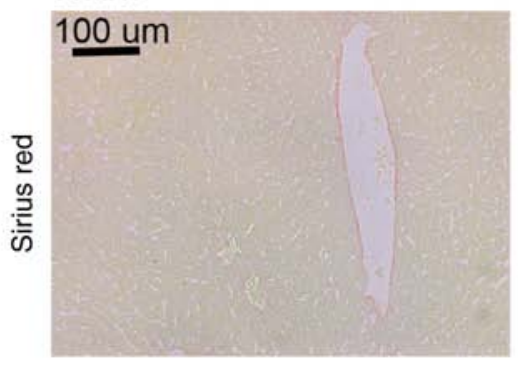

$\mathrm{CCl} 4$

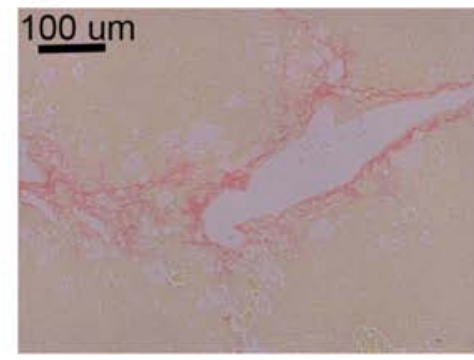

$\mathrm{CCl} 4$

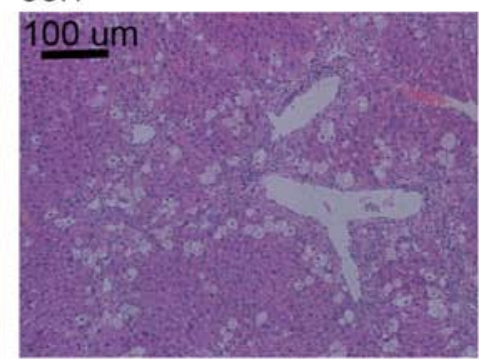

8 weeks

D

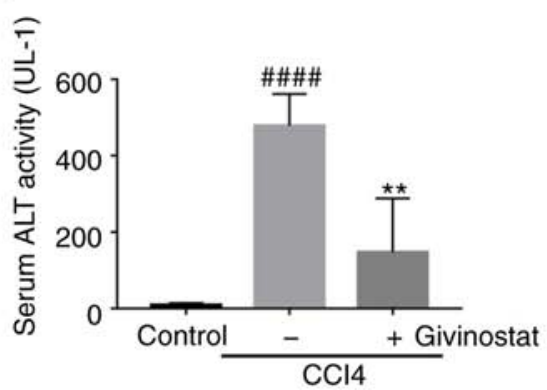

Solvent

$\mathrm{CCl} 4$ twice a week

$\mathrm{CCl} 4$ twice a week+ givinostat $10 \mathrm{mg} / \mathrm{kg} /$ day

CCl4+givinostat

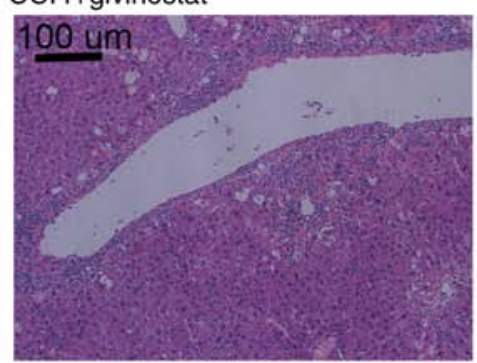

CCl4+givinostat
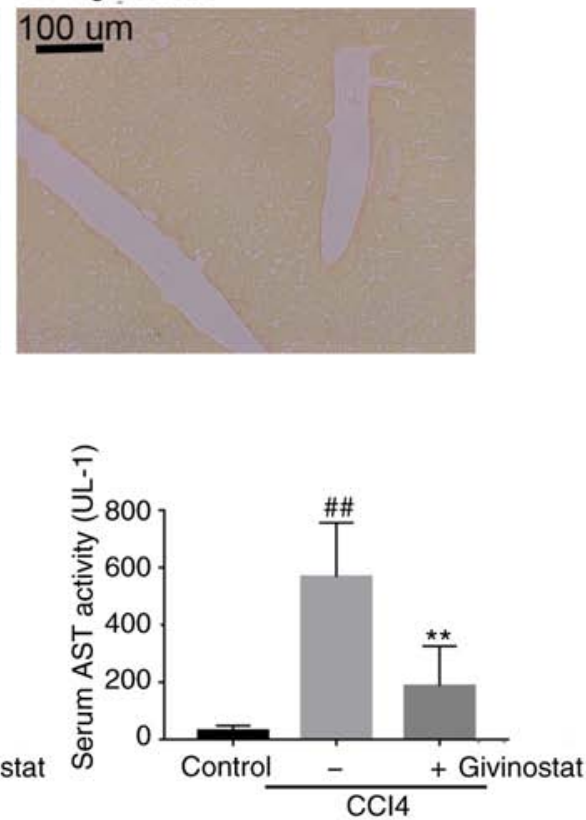

Figure 2. Givinostat treatment alleviates chronic liver injury and fibrosis in CCl4-induced mice. (A) Mice were randomly divided into the following three groups (n=8/group): Normal control, $\mathrm{CCl} 4$ and $\mathrm{CCl} 4$ + givinostat. In the $\mathrm{CCl} 4+$ givinostat group, mice were stimulated with $\mathrm{CCl} 4$ for 8 weeks, and in the last 6 weeks, the animals received an injection of givinostat $(10 \mathrm{mg} / \mathrm{kg} /$ day, i.p.). In the CCl4 group, mice were i.p. injected with CCl4 twice a week for 8 weeks. (B) Representative images of liver tissues, and microphotograph of hematoxylin and eosin-stained paraffin-embedded sections of liver tissues. Scale bar, $100 \mu \mathrm{m}$. (C) Representative microphotographs and quantifications of Sirius Red-stained paraffin-embedded sections of liver tissues by Image-Pro Plus 6.0 ( $\mathrm{n}=6-8$ images/group from normal control, CCl4 and CCl4 + givinostat groups). Scale bar, $100 \mu \mathrm{m}$. (D) Serum ALT and AST levels in mice from the normal control, $\mathrm{CCl} 4$ and $\mathrm{CCl} 4+$ givinostat groups ( $\mathrm{n}=6-8$ mice/group). Data are expressed as the mean $\pm \mathrm{SD}(\mathrm{n}=8) .{ }^{* *} \mathrm{P}<0.01,{ }^{* * * *} \mathrm{P}<0.0001 \mathrm{vs}$. the $\mathrm{CCl} 4$ group; ${ }^{\# \#} \mathrm{P}<0.01,{ }^{\# \# \# \#} \mathrm{P}<0.0001$ vs. the normal control group. CCl4, carbon tetrachloride; i.p., intraperitoneally; ALT, alanine aminotransferase; AST, aspartate aminotransferase.

in the present study, which investigated its potential role in the treatment of chronic liver fibrotic diseases. A widely used mouse liver fibrosis model with i.p. repeated injection of $\mathrm{CCl} 4$ was used to evaluate the efficacy of givinostat for the treatment of hepatic injury and liver fibrosis (29). C57BL/6J mice were i.p. injected with $\mathrm{CCl} 4$ for 2 weeks; at that time there was already mild liver fibrosis (30). Mice were then treated with givinostat for the following 6 weeks after $\mathrm{CCl} 4$ treatment
(Fig. 2A). As shown in Fig. 2B, persistent i.p. injection of $\mathrm{CCl} 4$ for 8 weeks caused hepatocyte steatosis (Fig. 2B middle panel), varying degrees of central venous wall thickening and collagen deposition (Fig. 2C middle panel), which are consistent with previous reports $(31,32)$. The results indicated that givinostat treatment markedly ameliorated the extent of hepatocyte steatosis (Fig. 2B right panel). Since the deposition of ECM (primarily collagens) is the major characteristic 

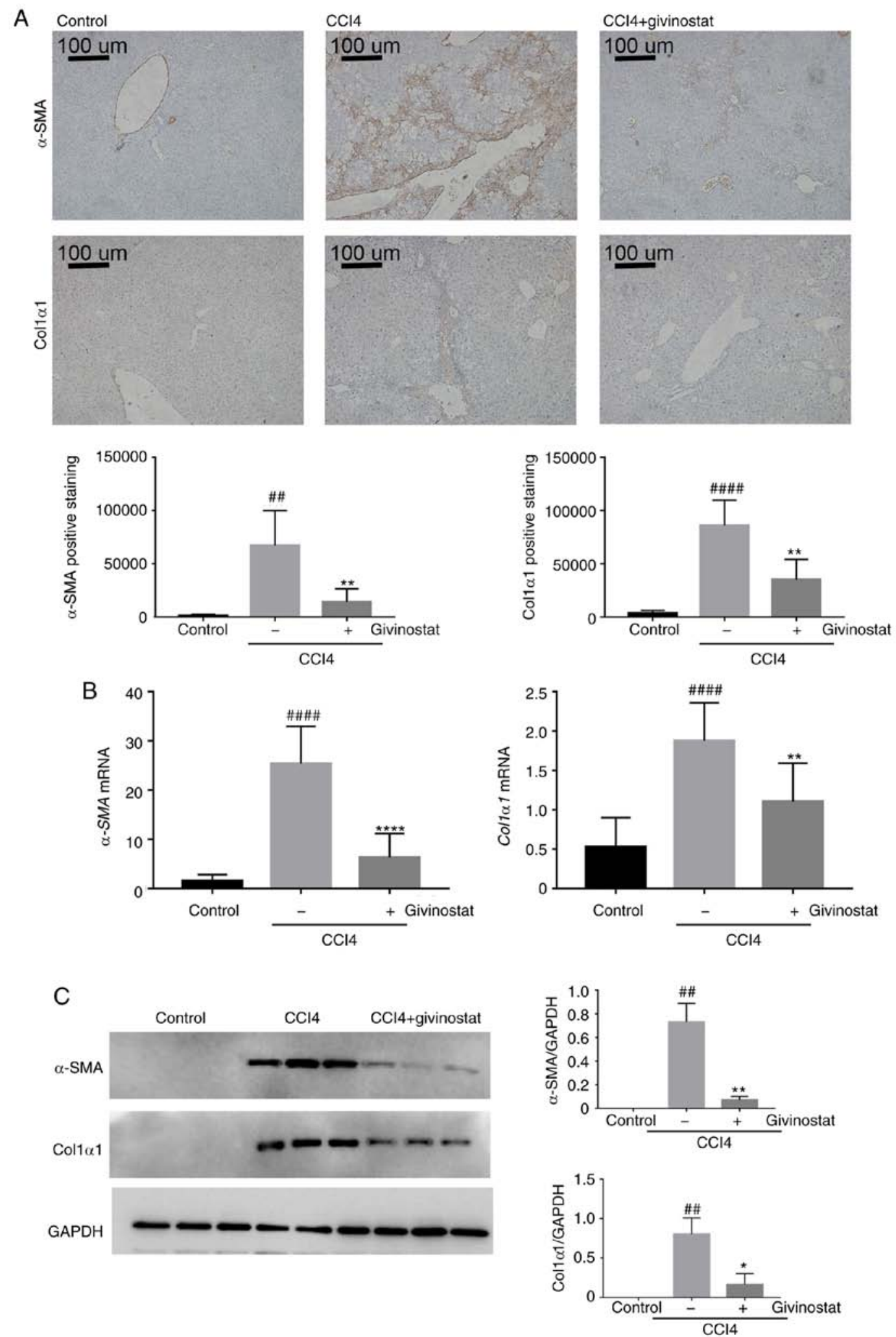

Figure 3. Givinostat inhibits hepatic stellate cell activation in CCl4-induced mice. (A) Representative images and quantifications of immunohistochemical staining for $\alpha$-SMA and Coll $\alpha 1$ of liver sections ( $\mathrm{n}=6-8 \mathrm{images} /$ group from normal control, CCl4 and CCl4 + givinostat groups). Scale bar, $100 \mu \mathrm{m}$. (B) Reverse transcription-quantitative PCR analysis of the fibrosis markers $\alpha$-SMA and Coll $\alpha 1$ (n=6-8/group from normal control, CCl4 and CCl4 + givinostat groups). (C) Western blot analysis and semi-quantification of $\alpha$-SMA and Coll $\alpha 1$ protein levels in mouse liver tissues. Data are expressed as the mean \pm SD $(n=6-8)$. ${ }^{*} \mathrm{P}<0.05,{ }^{* *} \mathrm{P}<0.01,{ }^{* * * *} \mathrm{P}<0.0001$ vs. the $\mathrm{CCl} 4$ group; ${ }^{\# \#} \mathrm{P}<0.01,{ }^{\# \# \# "} \mathrm{P}<0.0001$ vs. the normal control group. CCl4, carbon tetrachloride; $\alpha$-SMA, $\alpha$-smooth muscle actin; Col1 $\alpha 1$, collagen type I.

of liver fibrosis, the collagen contents were evaluated based on Sirius Red staining. Images ( $n=6-8$ images per group) showed that hepatic lobules maintained a normal physiological structure in the liver of control mice, while higher collagen accumulation was observed in the liver of CCl4-treated mice $(\mathrm{P}<0.0001$; Fig. $2 \mathrm{C})$. In the givinostat treatment group, the 
hepatic structure was improved and collagen was decreased $(\mathrm{P}<0.0001$; Fig. 2C). There was a significant increase in the levels of serum ALT $(n=6 ; 469 \pm 41.65 ; \mathrm{P}<0.0001)$ and AST $(\mathrm{n}=6 ; 536.8 \pm 95.53 ; \mathrm{P}<0.01)$ in $\mathrm{CCl}$-treated mice, whereas treatment with givinostat significantly decreased these serum markers of liver injury, ALT $(n=6 ; 137.7 \pm 71.72 ; \mathrm{P}<0.01)$ and AST ( $=6 ; 156.5 \pm 71.44 ; \mathrm{P}<0.01)$ (Fig. 2D). Furthermore, no systemic toxicity was observed at the dose of givinostat used in this experiment (data not shown). Thus, givinostat treatment significantly alleviated liver fibrosis and injury in vivo.

Givinostat inhibits HSC activation in mice with CCl4-induced liver fibrosis. Quiescent HSCs are activated on account of liver injury and considered to be the primary source of ECM yielding during hepatic fibrosis. Since givinostat significantly inhibited HSC activation in vitro and alleviated liver fibrosis in vivo, the present study assessed whether it inhibited HSC activation in vivo. $\alpha$-SMA and Coll $\alpha 1$ are the most abundant ECM proteins in liver tissue, and are markers of HSC activation $(33,34)$. Thus, $\alpha$-SMA or Coll $\alpha 1$-positive cells were detected by morphometric quantification to evaluate the accumulation of activated HSCs in mouse liver tissues. Immunohistochemical staining for $\alpha$-SMA $(\mathrm{n}=6 ; 65.800 \pm 14.861 ; \mathrm{P}<0.01)$ or Coll $\alpha 1(\mathrm{n}=6 ; 8.215 \pm 1.069$; $\mathrm{P}<0.0001)$ showed that positively stained brown-colored cells were notably increased in the liver tissues of mice treated with CCl4 (Fig. 3A middle panel). By contrast, immunohistochemical staining for $\alpha-S M A(n=6 ; 12.886 \pm 5.603 ; \mathrm{P}<0.01)$ or Coll $\alpha 1(\mathrm{n}=6 ; 3.140 \pm 859.9 ; \mathrm{P}<0.01)$ in the liver tissue of givinostat-treated mice was much weaker compared with that of solvent-treated mice, almost at a level similar to that of the normal control group (Fig. 3A right panel). RT-qPCR analysis confirmed that the increase in mRNA expression of $\alpha-S M A$ and Coll $\alpha 1$ in the liver tissues of $\mathrm{CCl} 4$-challenged mice was significantly reduced by givinostat treatment $(\mathrm{P}<0.01$; Fig. $3 \mathrm{~B})$. Consistently, western blot analysis further confirmed that the protein expression levels of $\alpha$-SMA and Coll $\alpha 1$ in mouse liver tissues were increased in the CCl4-chanlleged group, and were reduced by givinostat treatment $(\mathrm{P}<0.05$; Fig. $3 \mathrm{C})$. Taken together, these results demonstrated that givinostat alleviated liver fibrosis and inhibited HSC activation in vivo.

Identification of crucial genes for HSC activation that are regulated by givinostat via transcriptomic analysis. To explore the mechanism underlying the improvement of hepatic fibrosis by givinostat treatment in CCl4-challenged mice, RNA-seq analysis was performed to compare the gene expression profile of liver tissues from $\mathrm{CCl} 4$-challenged mice with or without givinostat treatment. Differential gene expression analysis identified genes upregulated or downregulated in givinostat-treated group compared with their expression in the solvent group in CCl4-challenged mice. The most significantly regulated genes by givinostat treatment are shown in Fig. 4A. RT-qPCR analysis confirmed that givinostat treatment inhibited or upregulated the mRNA expression of these genes in liver tissues (Fig. 4B), which was consistent with the RNA-seq results.

The present study next examined whether givinostat regulated the transcription of these genes in vitro in HSCs. The mRNA expression of these genes was analyzed via RT-qPCR in HSC LX- 2 cells stimulated by TGF- $\beta 1$ in the absence or presence of givinostat treatment. The mRNA expression levels of $M s l n, D m k n$ and $U p k 3 b$ were also reduced in HSC LX-2 cells (Fig. 4C), which was consistent with the findings in liver tissues of mice treated with givinostat. As for the genes that were most notably upregulated by givinostat treatment in vivo, their mRNA expression was increased in vitro to a much lower extent (Fig. 4C). As shown in Fig. 4D, givinostat dose-dependently inhibited $M s l n, D m k n$ and $U p k 3 b$ mRNA expression in the presence of TGF- $\beta 1$. These results confirmed that givinostat inhibited $M s l n, D m k n$ and $U p k 3 b$ gene expression in vivo and in vitro.

To further validate whether these givinostat-regulated genes play crucial roles during HSC activation, the effect of Msln, Dmkn and Upk3b depletion on TGF- $\beta 1$-induced HSC activation was examined. RT-qPCR analysis confirmed the knockdown efficiency of Msln, Dmkn and Upk3b by siRNA, which resulted in $>70 \%$ reduction in mRNA levels in HSC LX-2 cells compared with that of the siControl group $(\mathrm{P}<0.0001$; Fig. 5A). RT-qPCR analysis showed that the knockdown of Msln, Dmkn and Upk3b inhibited the mRNA levels of $\alpha-S M A$ and Coll 1 in HSC-LX2 cells stimulated with TGF- $\beta 1$ compared with the siControl + TGF- $\beta 1$ group $(\mathrm{P}<0.0001$; Fig. 5B and $\mathrm{C})$. These results demonstrated that givinostat inhibited $M s l n, D m k n$ and $U p k 3 b$ expression, and these genes were shown to be crucial for HSC activation.

\section{Discussion}

As a chronic hepatic disease with limited treatment options, liver fibrosis affects millions of individuals worldwide. Fibroblasts and myofibroblasts, which are primarily responsible for the synthesis of ECM proteins, have been identified as pivotal fibrotic effectors in multiple organs (35). HSCs, following activation and transformation to a myofibroblast phenotype, as marked by expression of $\alpha$-SMA, are the dominating cell type that produce ECM proteins during liver fibrogenesis $(36,37)$. Thus, the inactivation of HSCs is currently regarded as a potential treatment strategy for the treatment of hepatic fibrosis (21).

The present study established high-throughput screening assays to identify compounds that inhibited HSC activation. In view of the potential regulatory role of epigenetic mechanisms in modulating HSC function and reprogramming, an epigenetic inhibitor library was screened. The HDACi givinostat, which has been used in phase I/II clinical trials for Duchenne muscular dystrophy (15), was identified and validated as a potent inhibitor of HSC activation in vitro and in vivo. In the screening assay, givinostat was the most potent inhibitor of HSC activation, and it was more potent than other HDACi drugs. It was also observed that givinostat treatment alleviated liver fibrosis and liver injury in CCl4-injected mice, which had shown mild liver fibrosis. The liver organization architecture and function in mice with hepatic fibrosis were markedly improved after givinostat treatment. A previous study reported that givinostat can inhibit the proliferation and induce the apoptosis of HSC cells, thereby inhibiting liver fibrosis in mice subjected to a high-fat diet combined with intraperitoneal injection of CCl4 (38). However, ingestion of an obesogenic diet in that model could lead to the development 
A

\begin{tabular}{ll|l}
\hline \multicolumn{3}{c}{ Givinostat-treated vs CCI4-induced liver fibrosis model } \\
\hline Gene & Log2Fold-change & \\
Ms $/ n^{*}$ & -6.701855899 & \\
Dmkn* & -6.109074243 & \\
Upk3b* & -5.201986187 & \\
Upk1b & -4.35607744 & Inhibited by givinostat \\
Adgrd1 & -2.966815175 & \\
Cebpe & -2.110823927 & \\
Eif4ebp3 & 2.140357193 & \\
SIc2a5 & 2.150984153 & \\
Sult3al & 2.458027153 & \\
E030018b13rik & 2.835231844 & Upregulated by givinostat \\
Snai3 & 2.874938114 & \\
Ntrk1 & 3.183048653 & \\
\hline
\end{tabular}
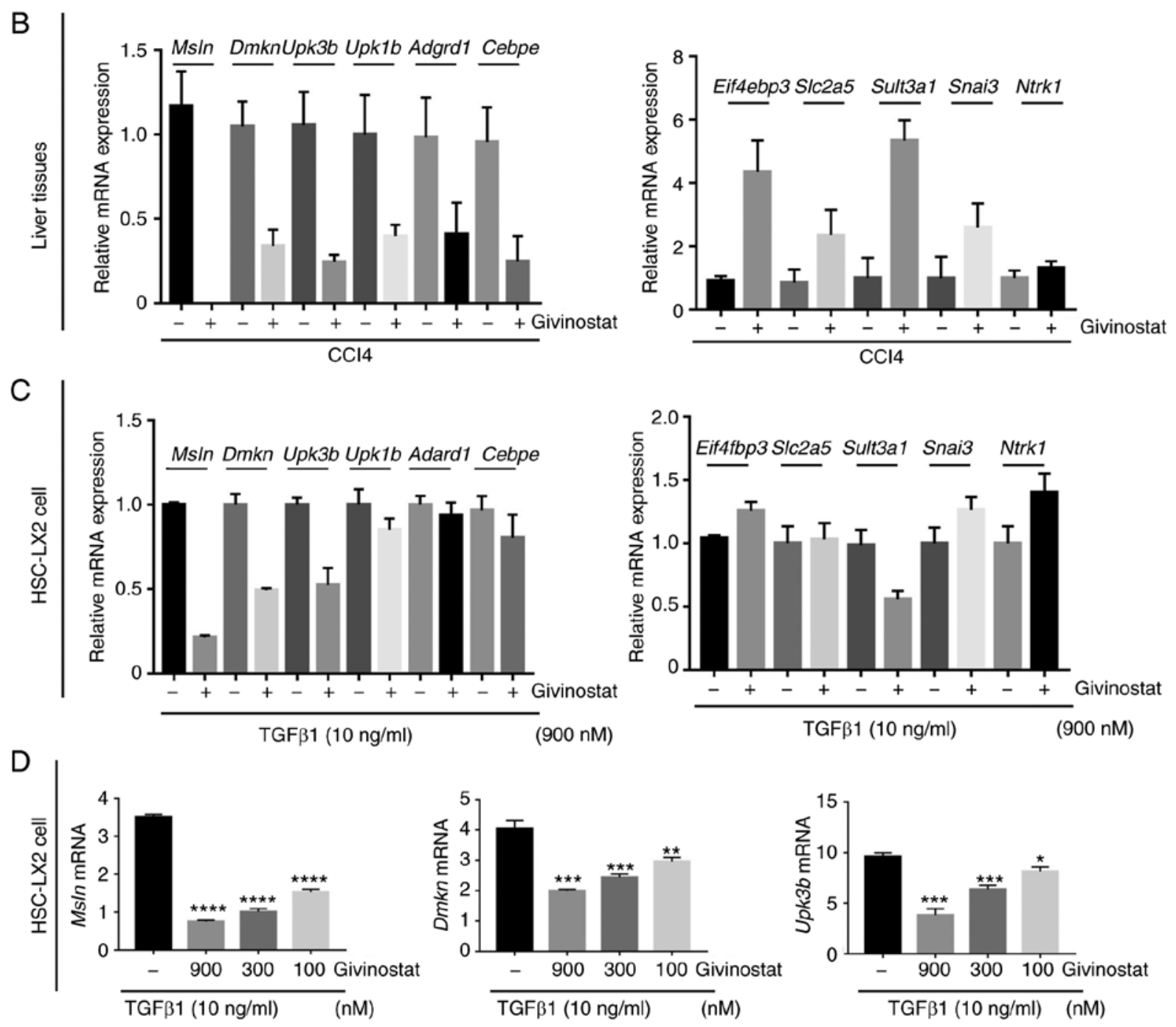

Figure 4. Identification of genes for HSC activation that are regulated by givinostat in vivo and in vitro via transcriptomic analysis. (A) RNA-sequencing analysis of liver tissue extracted from $\mathrm{CCl} 4(\mathrm{n}=4)$ and $\mathrm{CCl} 4$ + givinostat-treated mice $(\mathrm{n}=4)$. Diagram of the top genes with the most significant changes with adjusted $\mathrm{P}<0.05$ and absolute value of $\log 2$ fold-change $>1.5$. (B) RT-qPCR analysis was performed to validate the expression of the genes exhibiting major changes following givinostat treatment in vivo in liver tissues $(n=4)$. (C) RT-qPCR analysis was performed to validate the expression of the genes displayed in panel (B) in HSC LX-2 cells treated with givinostat in the presence of TGF- $\beta 1$. (D) RT-qPCR analysis of the expression of three genes in HSC LX-2 cells treated with different concentrations of givinostat. The results are normalized to $\beta$-actin and error bars indicate the SD of three independent experiments. Data are expressed as the mean \pm SD. ${ }^{*} \mathrm{P}<0.05,{ }^{* *} \mathrm{P}<0.01,{ }^{* * *} \mathrm{P}<0.001,{ }^{* * * * *} \mathrm{P}<0.0001$ vs. the TGF- $\beta 1$ group. HSC, hepatic stellate cell; RT-qPCR, reverse transcription-quantitative PCR; CCl4, carbon tetrachloride; Dmkn, dermokine; Msln, mesothelin; Upk3b, uroplakin-3b. 
A

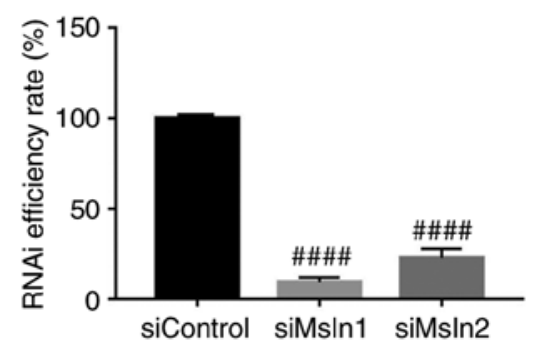

B
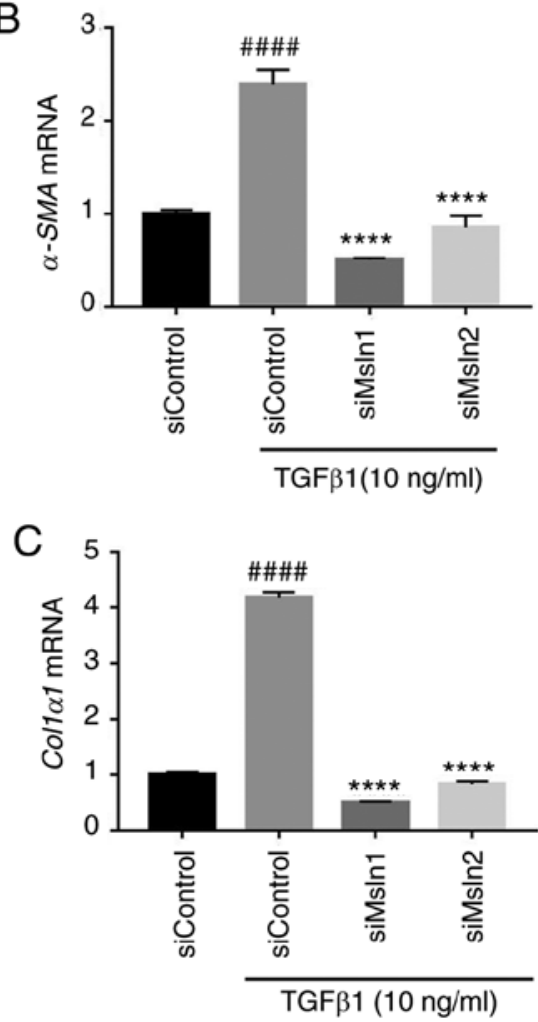
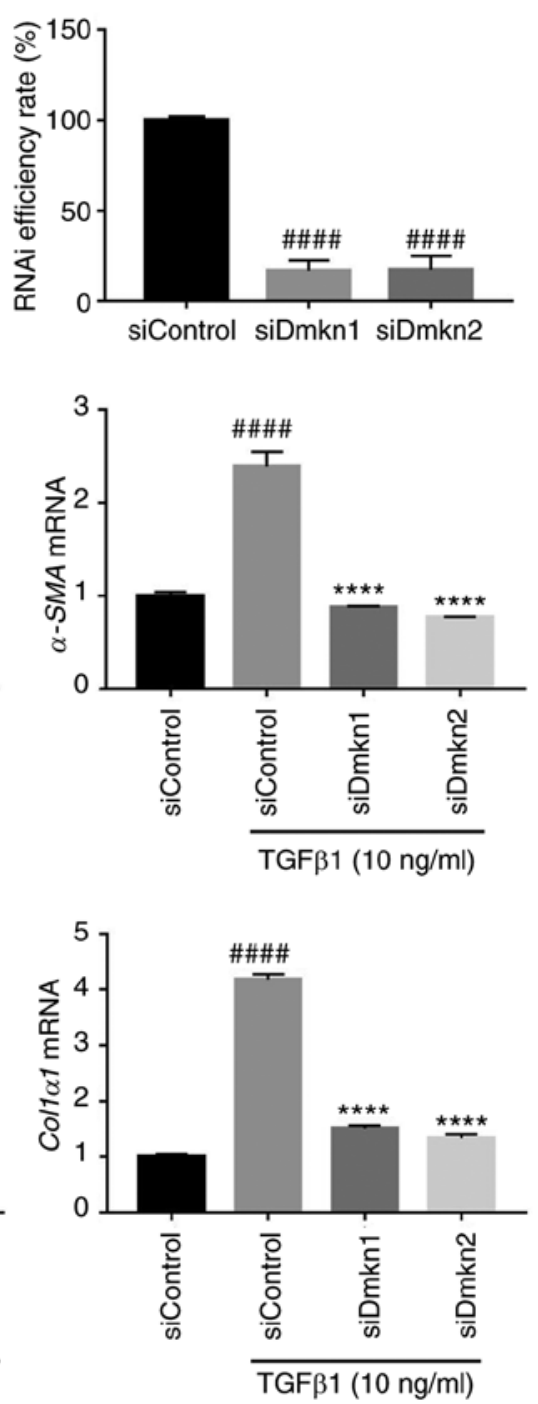
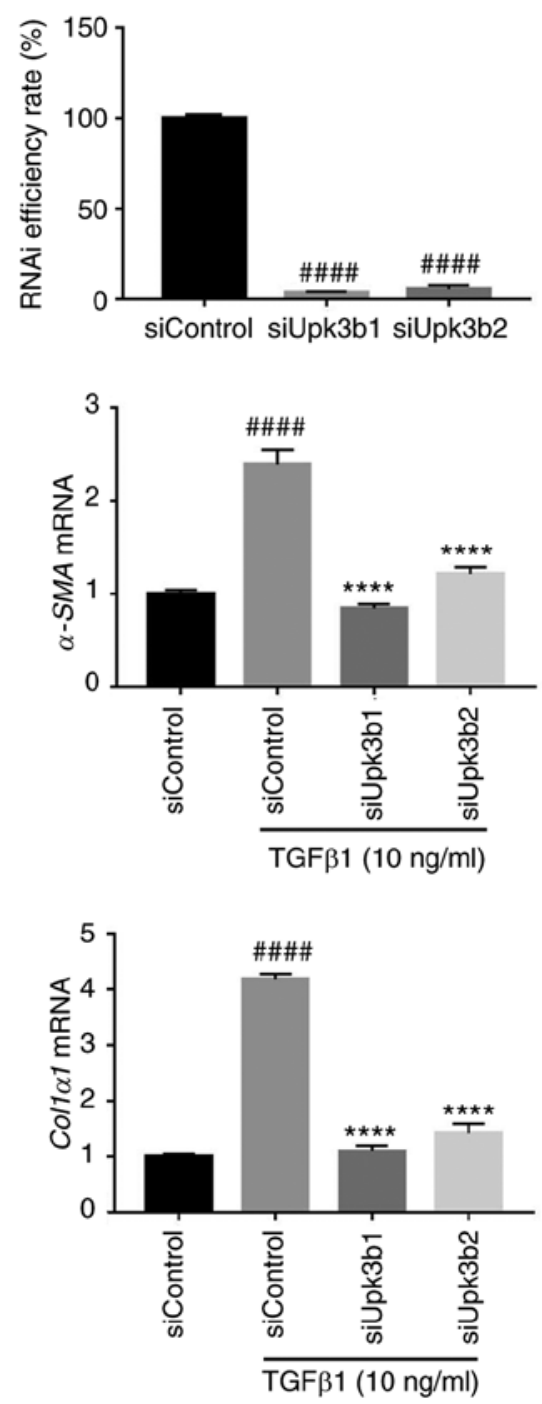

Figure 5. Msln, Dmkn and $U p k 3 b$ knockdown significantly inhibits the activation of HSCs. (A) Knockdown efficiency of $M s l n, D m k n$ and $U p k 3 b$ by siRNA in HSC LX-2 cells. The mRNA expression of fibrogenic genes (B) $\alpha$-SMA and (C) Coll $\alpha 1$ were quantified by reverse transcription-quantitative PCR in HSC LX-2 cells transfected with siRNA (siControl, siMsln, siDmkn and siUpk $3 b$ ). The results are normalized to $\beta$-actin, and error bars indicate the SD of three

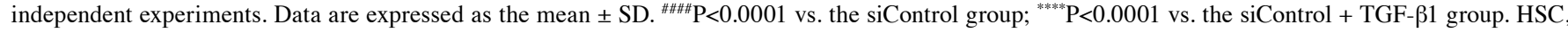
hepatic stellate cell; $\alpha$-SMA, $\alpha$-smooth muscle actin; Coll $\alpha 1$, collagen type I; Dmkn, dermokine; Msln, mesothelin; Upk3b, uroplakin-3b; si-, small interfering RNA; RNAi, RNA interference.

of steatosis, which might result in steatohepatitis and progressive fibrosis (16); thus, the alleviation of liver fibrosis might also be derived from the reduction of steatosis by givinostat.

To evaluate the effects of givinostat on a CCl4-induced liver fibrosis model, the present study showed a reduction in HSC activation and alleviation of liver fibrosis by givinostat in vivo, and also ruled out the possibility that the alleviation of fibrosis by givinostat was due to reduced steatosis. It has also been reported that HDAC9 knockdown can inhibit HSC activation and decrease fibrogenic gene expression in HSC LX-2 cells (39). Moreover, in agreement with the anti-fibrotic effects of givinostat shown in the present study, givinostat was reported to decrease endothelial-to-mesenchymal transition and reduce cardiac fibrosis, leading to improved heart performance and protection of blood vessels from apoptosis in a mouse model of acute myocardial injury (19). Moreover, single or repeated oral administration of givinostat in humans has been found to be safe, as shown in a previous clinical trial (29). In the present mouse model, no systemic toxicity was observed at the dose of givinostat used in this experiment. Thus, the present study suggested that givinostat inhibited hepatic fibrosis and HSC activation in vivo, and raised the possibility that an existing drug can be repurposed as a new treatment of hepatic fibrosis.

Transcriptomic analysis revealed the most significantly regulated genes in the givinostat treatment group in comparison with the solvent group, among which, Dmkn, Msln and $U p k 3 b$ were validated in vitro in HSC LX-2 cells as crucial genes regulating HSC activation. When Msln, Dmkn or Upk3b expression was knocked down, the increased mRNA expression of $\alpha-S M A$ and Coll $\alpha 1$ in response to TGF- $\beta 1$ stimulation was significantly reduced in HSC LX-2 cells, suggesting that these three genes may play crucial roles in the activation of HSCs. To the best of our knowledge, the role of Msln, Dmkn and Upk3b in HSC activation was reported for the first time in the present study. Moreover, givinostat treatment significantly reduced the mRNA expression of Dmkn, Msln and 
$U p k 3 b$ in both a mouse model and HSC-LX2 cells. Certain genes that were significantly affected by givinostat treatment in vivo were not affected in vitro in HSC LX-2 cells, which may be unrelated to HSC activation or could be the result of other cell types in the liver, such as endothelial, Kupffer and bile-duct cells $(40,41)$. Thus, the identification of givinostat as an inhibitor of HSC activation and its use as a chemical probe led to the identification of novel regulators of HSC activation.

In summary, the present study established a high-throughput cell-based assay for the identification of a compound targeting HSC activation, and identified givinostat as a potent inhibitor of HSC activation in vitro and in vivo. Novel regulators of HSC activation were identified using givinostat as a probe, and these findings illustrated the efficacy of an epigenetic strategy that targets HSC activation for the treatment of hepatic fibrosis.

\section{Acknowledgements}

Not applicable.

\section{Funding}

The present study was financially supported by the National Natural Science Foundation of China (grant nos. 81070344, 81803554, 91853205, 81625022, 81821005 and 81773568), The Ministry of Science and Technology of China (grant no. 2015CB910304), The National Science \& Technology Major Project of China (grant no. 2018ZX09711002) and Youth Innovation Promotion Association (grant no. 2017333).

\section{Availability of data and materials}

The datasets generated and/or analyzed during the current study are available in the GEO repository, https://www.ncbi. nlm.nih.gov/geo/query/acc.cgi?acc=GSE161981. The datasets used and/or analyzed during the current study are available from the corresponding author on reasonable request.

\section{Authors' contributions}

HMH, YJL, LPL, LY and JJP performed the immunofluorescence staining, western blotting, siRNA transfection and mouse liver fibrosis experiments, analyzed the corresponding data and wrote the manuscript. XRZ, SJF and JH contributed to manuscript writing and modification and analyzed the RNA-seq data. GML, CL, CCS and YYZ conceived and supervised the project, and revised the manuscript. The present article was conducted in accordance with the ARRIVE guideline checklist. The authors are accountable for all aspects of the work in ensuring that questions related to the accuracy or integrity of any part of the work are appropriately investigated and resolved. HMH, XRZ and LPL confirm the authenticity of all the raw data. All authors read and approved the final manuscript.

\section{Ethics approval and consent to participate}

Animal care was carried out according to the guidelines of the Principles of Laboratory Animal Care, and the protocol was approved by the Institute Animal Care and Use Committee at the Shanghai Institute of Materia Medica (approval no. 2018-12-LC-11; Shanghai, China).

\section{Patient consent for publication}

Not applicable.

\section{Competing interests}

The authors declare that they have no competing interests.

\section{References}

1. Mokdad AA, Lopez AD, Shahraz S, Lozano R, Mokdad AH, Stanaway J, Murray CJ and Naghavi M: Liver cirrhosis mortality in 187 countries between 1980 and 2010: A systematic analysis. BMC Med 12: 145, 2014.

2. Schuppan D and Afdhal NH: Liver cirrhosis. Lancet 371: 838-851, 2008.

3. Bataller R and Brenner DA: Liver fibrosis. J Clin Invest 115 : 209-218, 2005.

4. Weiskirchen R and Tacke F: Liver fibrosis: From pathogenesis to novel therapies. Dig Dis 34: 410-422, 2016.

5. Ginès P, Cárdenas A, Arroyo V and Rodés J: Management of cirrhosis and ascites. N Engl J Med 350: 1646-1654, 2004.

6. Bárcena C, Stefanovic M, Tutusaus A, Joannas L, Menéndez A, García-Ruiz C, Sancho-Bru P, Marí M, Caballeria J, Rothlin CV, et al: Gas6/Axl pathway is activated in chronic liver disease and its targeting reduces fibrosis via hepatic stellate cell inactivation. J Hepatol 63: 670-678, 2015.

7. Koh HB, Scruggs AM and Huang SK: Transforming growth factor- $\beta 1$ increases DNA methyltransferase 1 and 3a expression through distinct post-transcriptional mechanisms in lung fibroblasts. J Biol Chem 291: 19287-19298, 2016.

8. Mann J, Oakley F, Akiboye F, Elsharkawy A, Thorne AW and Mann DA: Regulation of myofibroblast transdifferentiation by DNA methylation and MeCP2: Implications for wound healing and fibrogenesis. Cell Death Differ 14: 275-285, 2007.

9. Watson CJ, Collier P, Tea I, Neary R, Watson JA, Robinson C, Phelan D, Ledwidge MT, McDonald KM, McCann A, et al: Hypoxia-induced epigenetic modifications are associated with cardiac tissue fibrosis and the development of a myofibroblast-like phenotype. Hum Mol Genet 23: 2176-2188, 2014.

10. Liu N, He S, Ma L, Ponnusamy M, Tang J, Tolbert E, Bayliss G, Zhao TC, Yan H and Zhuang S: Blocking the class I histone deacetylase ameliorates renal fibrosis and inhibits renal fibroblast activation via modulating TGF-beta and EGFR signaling. PLoS One 8: e54001, 2013.

11. Marumo T, Hishikawa K, Yoshikawa M, Hirahashi J, Kawachi S and Fujita T: Histone deacetylase modulates the proinflammatory and -fibrotic changes in tubulointerstitial injury. Am J Physiol Renal Physiol 298: F133-F141, 2010.

12. Tung CW, Hsu YC, Cai CJ, Shih YH, Wang CJ, Chang PJ and Lin CL: Trichostatin A ameliorates renal tubulointerstitial fibrosis through modulation of the JNK-dependent Notch-2 signaling pathway. Sci Rep 7: 14495, 2017.

13. Wu M, Lin P, Li L, Chen D, Yang X, Xu L, Zhou B, Wang C, Zhang Y, Luo C and Ye C: Reduced asymmetric dimethylarginine accumulation through inhibition of the type I protein arginine methyltransferases promotes renal fibrosis in obstructed kidneys. FASEB J 33: 6948-6956, 2019.

14. Peng J, Li J, Huang J, Xu P, Huang H, Liu Y, Yu L, Yang Y, Zhou B, Jiang H, et al: p300/CBP inhibitor A-485 alleviates acute liver injury by regulating macrophage activation and polarization. Theranostics 9: 8344-8361, 2019.

15. Tomaselli D, Lucidi A, Rotili D and Mai A: Epigenetic polypharmacology: A new frontier for epi-drug discovery. Med Res Rev 40: 190-244, 2020.

16. Tsuchida T, Lee YA, Fujiwara N, Ybanez M, Allen B, Martins S, Fiel MI, Goossens N, Chou HI, Hoshida Y and Friedman SL: A simple diet- and chemical-induced murine NASH model with rapid progression of steatohepatitis, fibrosis and liver cancer. J Hepatol 69: 385-395, 2018. 
17. Scholten D, Trebicka J, Liedtke $\mathrm{C}$ and Weiskirchen R: The carbon tetrachloride model in mice. Lab Anim 49 (1 Suppl): S4-S11, 2015.

18. National Research Council (US) Committee for the Update of the Guide for the Care and Use of Laboratory Animals. Guide for the Care and Use of Laboratory Animals. 8th edition. Washington (DC): National Academies Press, Washington (DC), 2011.

19. Milan M, Pace V, Maiullari F, Chirivì M, Baci D, Maiullari S, Madaro L, Maccari S, Stati T, Marano G, et al: Givinostat reduces adverse cardiac remodeling through regulating fibroblasts activation. Cell Death Dis 9: 108, 2018.

20. Lan T, Li C, Yang G, Sun Y, Zhuang L, Ou Y, Li H, Wang G, Kisseleva T, Brenner D and Guo J: Sphingosine kinase 1 promotes liver fibrosis by preventing miR-19b-3p-mediated inhibition of CCR2. Hepatology 68: 1070-1086, 2018.

21. Zhang K, Han X, Zhang Z, Zheng L, Hu Z, Yao Q, Cui H, Shu G, Si M, Li C, et al: The liver-enriched lnc-LFAR1 promotes liver fibrosis by activating TGF $\beta$ and Notch pathways. Nat Commun 8: 144, 2017.

22. Dobin A, Davis CA, Schlesinger F, Drenkow J, Zaleski C, Jha S, Batut P, Chaisson $\mathrm{M}$ and Gingeras TR: STAR: Ultrafast universal RNA-seq aligner. Bioinformatics 29: 15-21, 2013.

23. Liao Y, Smyth GK and Shi W: FeatureCounts: An efficient general-purpose program for assigning sequence reads to genomic features. Bioinformatics 30: 923-930, 2014.

24. Robinson MD, McCarthy DJ and Smyth GK: edgeR: A Bioconductor package for differential expression analysis of digital gene expression data. Bioinformatics 26: 139-140, 2010.

25. Jose AF: The Benjamini-Hochberg method in the case of discrete test statistics. Int J Biostat 3: 11, 2007.

26. Huang DW, Sherman BT and Lempicki RA: Systematic and integrative analysis of large gene lists using DAVID Bioinformatics Resources. Nat Protoc 4: 44-57, 2009.

27. Shannon P, Markiel A, Ozier O, Baliga NS, Wang JT, Ramage D, Amin N, Schwikowski B and Ideker T: Cytoscape: A software environment for integrated models of biomolecular interaction networks. Genome Res 13: 2498-2504, 2003.

28. Livak KJ and Schmittgen TD: Analysis of relative gene expression data using real-time quantitative PCR and the 2(-Delta Delta C(T)) method. Methods 25: 402-408, 2001.

29. Pan RL, Xiang LX, Wang P, Liu XY, Nie L, Huang W and Shao JZ: Low-molecular-weight fibroblast growth factor 2 attenuates hepatic fibrosis by epigenetic down-regulation of Delta-like1. Hepatology 61: 1708-1720, 2015.

30. Suh YG, Kim JK, Byun JS, Yi HS, Lee YS, Eun HS, Kim SY, Han KH, Lee KS, Duester G, et al: $\mathrm{CD}_{11 b^{+}} \mathrm{Grl}^{+}$bone marrow cells ameliorate liver fibrosis by producing interleukin-10 in mice. Hepatology 56: 1902-1912, 2012.
31. Satishchandran A, Ambade A, Rao S, Hsueh YC, Iracheta-Vellve A, Tornai D, Lowe P, Gyongyosi B, Li J, Catalano D, et al: MicroRNA 122, regulated by GRLH2, protects livers of mice and patients from ethanol-induced liver disease. Gastroenterology 154: 238-252.e7, 2018.

32. King A, Houlihan DD, kavanagh D, Haldar D, Luu N, Owen A, Suresh S, Than NN, Reynolds G, Penny J, et al: Sphingosine-1-phosphate prevents egress of hematopoietic stem cells from liver to reduce fibrosis. Gastroenterology 153: 233-248.e16, 2017.

33. Han CY, Koo JH, Kim SH, Gardenghi S, Rivella S, Strnad P, Hwang SJ and Kim SG: Hepcidin inhibits Smad3 phosphorylation in hepatic stellate cells by impeding ferroportin-mediated regulation of Akt. Nat Commun 7: 13817, 2016.

34. Jia Y, Wang F, Guo Q, Li M, Wang L, Zhang Z, Jiang S, Jin H, Chen A, Tan S, et al: Curcumol induces RIPK1/RIPK3 complex-dependent necroptosis via JNK1/2-ROS signaling in hepatic stellate cells. Redox Biol 19: 375-387, 2018.

35. Hinz B, Phan SH, Thannickal VJ, Galli A, Bochaton-Piallat ML and Gabbiani G: The myofibroblast: One function, multiple origins. Am J Pathol 170: 1807-1816, 2007.

36. Mederacke I, Hsu CC, Troeger JS, Huebener P, Mu X, Dapito DH, Pradere JP and Schwabe RF: Fate tracing reveals hepatic stellate cells as dominant contributors to liver fibrosis independent of its aetiology. Nat Commun 4: 2823, 2013.

37. Friedman SL: Hepatic stellate cells: Protean, multifunctional, and enigmatic cells of the liver. Physiol Rev 88: 125-172, 2008.

38. Wang YG, Xu L, Wang T, Wei J, Meng WY, Wang N and Shi M: Givinostat inhibition of hepatic stellate cell proliferation and protein acetylation. World J Gastroenterol 21: 8326-8339, 2015.

39. Yang Y, Bae M, Park YK, Lee Y, Pham TX, Rudraiah S, Manautou J, Koo SI and Lee JY: Histone deacetylase 9 plays a role in the antifibrogenic effect of astaxanthin in hepatic stellate cells. J Nutr Biochem 40: 172-177, 2017.

40. Seki E, De Minicis S, Osterreicher CH, Kluwe J, Osawa Y, Brenner DA and Schwabe RF: TLR4 enhances TGF-beta signaling and hepatic fibrosis. Nat Med 13: 1324-1332, 2007.

41. Ramzy MM, Abdelghany HM, Zenhom NM and El-Tahawy NF: Effect of histone deacetylase inhibitor on epithelial-mesenchymal transition of liver fibrosis. IUBMB Life 70: 511-518, 2018

This work is licensed under a Creative Commons Attribution-NonCommercial-NoDerivatives 4.0 International (CC BY-NC-ND 4.0) License. 